\title{
Mitigating Inter-Symbol Interference using Blind Equalizer in a Wireless Communication System
}

\author{
Nadeem Ullah Khan \\ ICT, UET Peshawar \\ Pakistan
}

\author{
Aftab Hussain, $\mathrm{PhD}$ \\ Associate Professor, SPIT \\ Pakistan
}

\author{
Mudassar Nawaz \\ PTCL, Islamabad \\ Pakistan
}

\begin{abstract}
In modern world communication where multiple signal samples are transmitted at a time, a problematic phenomenon occurs called Inter symbol interference (ISI). In this phenomenon samples are affected by previous and next samples. This happens due to difference in the propagation delays over same path because of which symbols interact with each other at multiple points and over all signal is disturbed. Band limitation and multipath propagations are key issues of this phenomenon. Transmitted signal already contains white noise etc. The signal is affected by additive white noise as well. A suitable technique for reducing this ISI effect is adaptive method. Due to varying nature of noise detection, adaptive behavior of equalizer is necessary for effective mitigation. Adaptive algorithm requires a known training sequence to track time varying characteristics of the channel. Here the characteristics of channel are point of focus for the equalizers. Blind algorithms have a concept that they track time varying properties of channel as consequence of limitation in training sequence.
\end{abstract}

The paper deals with the designing of equalizers using different modulation techniques. Results are applied over two wireless channels using three different wireless access techniques. The equalizer works as a blind equalizer since it treats the signals without any training pattern thus making this idea innovative. A comprehensive analysis based results are inferred to ensure the proper equalization model to reduce the ISI effect.

\section{Keywords}

ISI, Blind Equalization, QAM, Rayleigh, Rician, QPSK, Equalizers, CMA, MMA, SCA, BPSK, QPSK, Convergence

\section{INTRODUCTION}

Adaptive method for mitigation of ISI is very common. Due to varying nature of noise, detection and adaptive behavior of equalizer based on detection process is necessary for effective mitigation. Adaptive algorithm requires a known training sequence to track time varying characteristics of channel. Here the characteristics of channel are point of focus for the equalizers. Blind algorithms have a concept that they track time varying properties of channel despite deficiency of training sequence [15].

Adaptive trained channel equalization initially developed by Lucky was used in landline telephone systems [20]. Since the systems were simple and there was a little clearance for a transmitted signal to get distorted therefore simple methodology was employed. In 1965 zero forcing (ZF) method was used in Finite Impulse Response (FIR) equalizers. Since the channel was approximately noiseless the channel had inverse zero forcing (ZF). Hoff and Widrow made another interesting technique later known a Least Mean Square (LMS) method in 1960 [21]. The Mean Square Error was reduced with this technique. After the signal eye opens the equalizer worked in tracking mode. This is also called as direction directed mode. The supervision process that was introduced was effective but it depended upon the initial condition of the coefficient of the equalizer.

Blind equalization does not need desired signal for the receiver. The channel and its input are not known and the main aim of blind equalizer is the recovery of an input sequence not known to the receiver. The calculations are based on probabilistic and statistical properties of the input sequence.

Based on statistical properties of the input data and sample signal, blind equalization inverts the channel. The spectra of the output and input of a channel is used to discover impulse response of a channel. With the help of the said procedure minimum phase channel can be shown. But the problem here is that most of the channels do not have minimum phase. A non-Gaussian sequence is needed along with nonlinear processing of the signal to identify non-minimum phase channel [30]. It can be said that there are two main properties of identification of minimum phase:

\section{- Using statistics of the retrieved signal.}

- Optimization of Cost function.

Design of cost function of equalizer is subjective rather objective. Most of the cost functions are intuitive rather objectively achieved. Due to this many possibility can overwhelm the calculations. Keeping in view this possibility there are calculation techniques for cost functions.

In this paper, random signal will be generated by using signal constellation and the modulation scheme used will be PSK and QAM. Doppler shift noise in Rician channel over $10 \mathrm{~Hz}$ will be imposed. The AWGN from -10 to $-20 \mathrm{~dB}$ is also introduced in it. Note that performance analysis of equalizer techniques for modulated signals is required to be understood before implementing it in a communication system. The channel affected by the above constants will be equalized through Least Mean Square (LMS) RLS initially to make research simple at first. The flow diagram for methodology is given as under.

Start $\rightarrow$ Signal Generation $\rightarrow$ Modulation $\rightarrow$ Inter Symbol Interference $\rightarrow$ Equalizer $\rightarrow$ Demodulation $\rightarrow$ Error Calculation and BER Plot $\rightarrow$ End

Note that random signals are generated with the use of signal constellation. Modulation is done using PSK and QAM schemes. Equalization essentially uses estimation of time dispersion which is the impulse response of the channel through which signal is transmitted. In traditional methods, a training signal is used to comprehend the channel which is varying. A regular check through training signal is used in this regard. For this to happen equalizer must be adaptable to 
update the system/equalizer settings as the time passes. The interval between two training signals is used for updating of the settings. Note the following before performing of system simulation in a typical environment. The cost function is calculated by time average of error and other factors.

\section{LITERATURE REVIEW}

In a research by Ding and $\mathrm{Li}$, performance comparison of blind channel equalization techniques was made [16]. An equalizer must maintain channel frequency response variation and cancelation multipath problems. They are also called echo-cancellers for the same reason. While convolving channel and equalizer impulse responses should stand equal that is null on opposite sample points inside the filter span and one at the center tap. In contrast with adaptive method, in place of training signal characteristics, transmitted signal characteristics are analyzed for the detection and mitigation process.

Ghadami and Moshirian discussed basic principles of blind channel equalization in which they gave fundamentals of blind channel equalization [17]. As blind channel, does not require pilot signals for the recovery of transmitted data, four diverse types of algorithms for the implementation of blind channel equalization were discussed. These were Least Means Square (LMS), Constant Modulus algorithm (CMA) etc. The mentioned techniques were pervasively being used for equalization. The conclusion on comparative analysis of CMA and LMS algorithms suggested that CMA performed slower than LMS algorithm.

In case of large value of noise CMA has better effects on the convergence rate. The results were based on D I Jones work named as 'A normalized constant modulus algorithm' [18]. Haykin suggested that conventional least mean square adaptive filters are implemented in receivers that are supervised [19]. By supervision or adaptive ability, it is meant that the equalizer provides the abilities of learning and inverting the distorting effects of the channel. Filter tap weights are used for calculations and enabling an equalizer with this feature. As vendor, specific training signals are different therefore implementation of a standard detector is difficult. That is why Blind Channel equalizers earned name on face of this problem.

Adaptive trained channel equalization initially developed by Lucky was used in landline telephone systems [20]. Since the systems were simple and there was a little clearance for a transmitted signal to get distorted therefore simple methodology was employed. In 1965 zero forcing (ZF) method was used in Finite Impulse Response (FIR) equalizers. Since the channel was approximately noiseless the channel had inverse zero forcing (ZF). Hoff and Widrow made another interesting technique later known a Least Mean Square (LMS) method in 1960 [21]. The Mean Square Error was reduced with this technique. After the signal eye opens the equalizer worked in tracking mode. This is also called as direction directed mode. The supervision process that was introduced was effective but it depended upon the initial condition of the coefficient of the equalizer.

Blind equalization does not need desired signal for the receiver. The channel and its input are not known and the main aim of blind equalizer is the recovery of an input sequence not known to the receiver. The calculations are based on probabilistic and statistical properties of the input sequence. This case was first discussed by Mazo and Allen [22]. The research proved in an analytical method that it was capable of inverting channel without the presence of training sequence. In 1975 Sato came up with an adaptive blind channel equalizer [23]. This equalizer was designed for PAM signals initially. Godrad in 1980 gave equalizers for more complex signals like V29, QAM and QPSK. Agee in 1983 gave results for AM/FM signals [24]. The main drawback of these early blind channel equalization techniques was the intuitive nature of the starting point.

Joint blind equalization and work on carrier phase was reported in 1984 by Goursat, Wesolowisky in 1987 [25]. Recent works on the same topic were done by Nandi and Abrar in 2010 [26]. These techniques were capable of recovery of true power of the transmitted data at convergence. This class of equalizers is called Bussgang-type. Bussgang equalization uses nonlinear estimate of the channel [27]. They are ISI (Inter symbol Interference) sensitive cost function which use higher order statistics.

The earliest comprehensive study of blind equalization problem was given by Ruget in 1980 [28]. If the signal is a non-Gaussian and identically distributed and noise is negligible, it is perfectly equalized. This result became the standard benchmark for equalizer to achieve. The research established a connection that higher order statistics of the channel are required for best possible equalization results. Sato algorithm later asserted the fact that MSE method should be used for better results so far as Blind Equalization is concerned. Shalvi in 1990 along with Weinstein gave condition of blind equalization [29]. Prior to the research of Shalvi, ensuring zero forcing was not compulsory. The zero forcing was not in common use in this method until the research of Shalvi.

\section{SUGGESTED SOLUTION}

Focusing on the mitigation of ISI effect, an innovative approach was developed to test the incoming signal using different modulation techniques over different wireless channels. BPSK, QPSK and QAM was used for Rayleigh and Rician Channels. Block diagram of the approach is shown in following figure 1 .

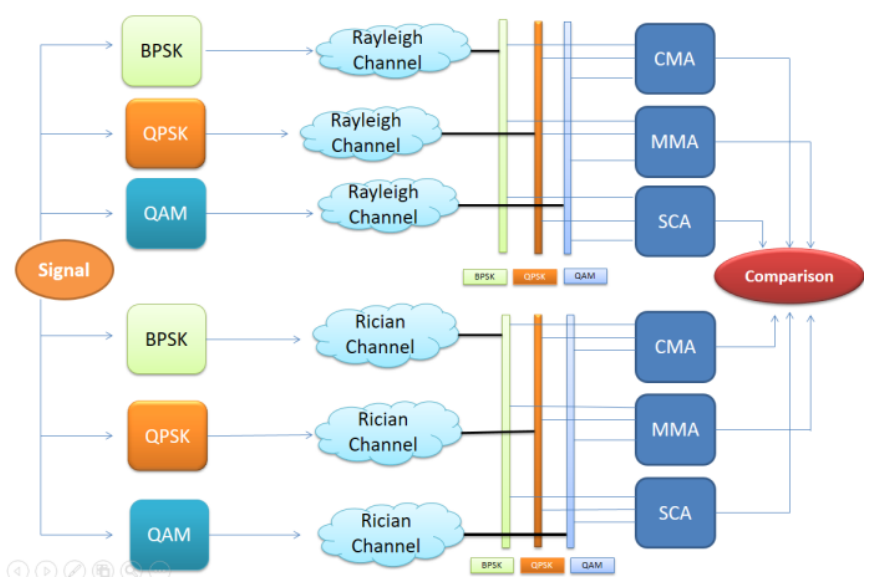

Figure 1: Model Approach

Incoming signal in figure 1 is applied a modulation technique with combination of a multiple access techniques over selected wireless channel and end results are compared. Results are compared based on the number of iterations used to perform the test and resulting errors. Iterations are the convergence rate of the signal showing the rate at which signal converged and dilated. Setup and results are discussed in next section. 


\section{SIMULATION RESULTS}

MATLAB was used to support the idea proposed. Using BPSK, QAM and QPSK following constellation points were utilized.

- $\quad$ BPSK $[-1,1]$,

- $\quad$ QPSK $[1-1, \mathrm{i}-\mathrm{i}]$

- $\quad$ QAM $[1+\mathrm{I}, 1-\mathrm{i},-1+\mathrm{i},-1-\mathrm{i}]$

Graphical results of various simulations carried over are shown below.

Figures 2-7 showing the results of single modulation techniques using CMA, MMA and SCA over Rayleigh and Rician channel.

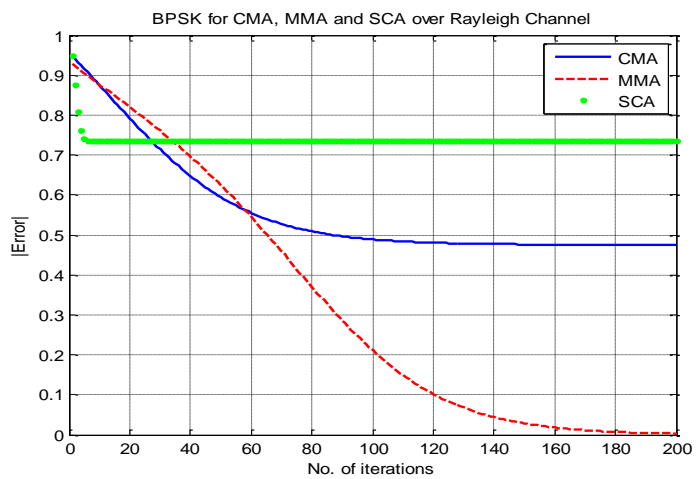

Figure 2: BPSK for CMA, SCA and MMA over Rayleigh Channel

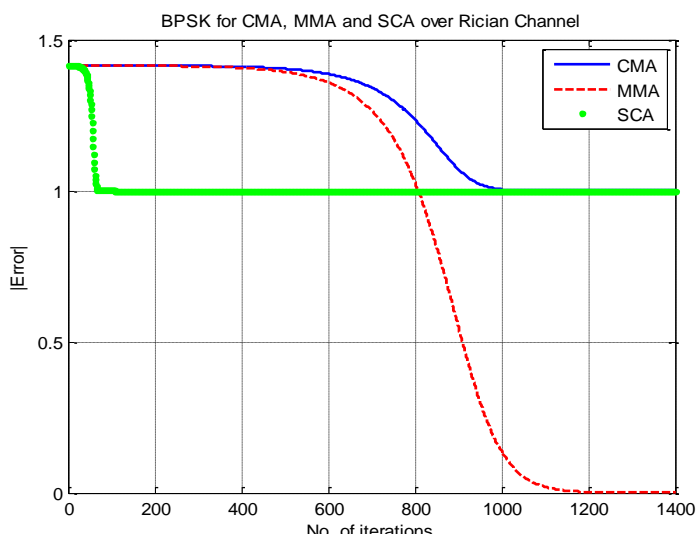

Figure 3: BPSK for CMA, SCA and MMA over Rician Channel

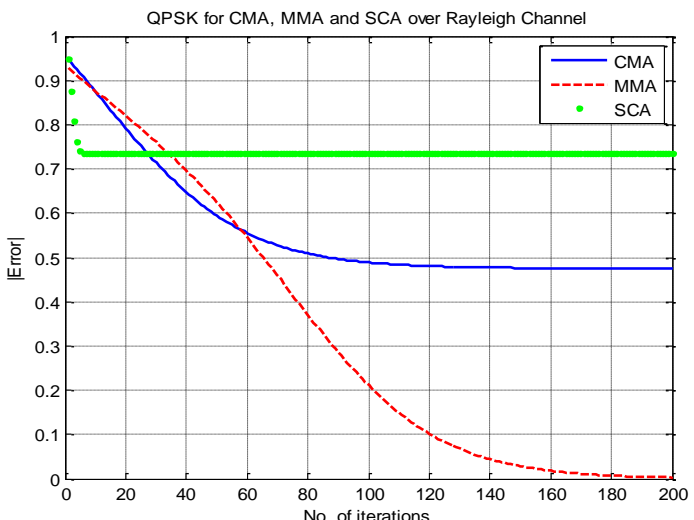

Figure 4: QPSK for CMA, SCA and MMA over Rayleigh Channel

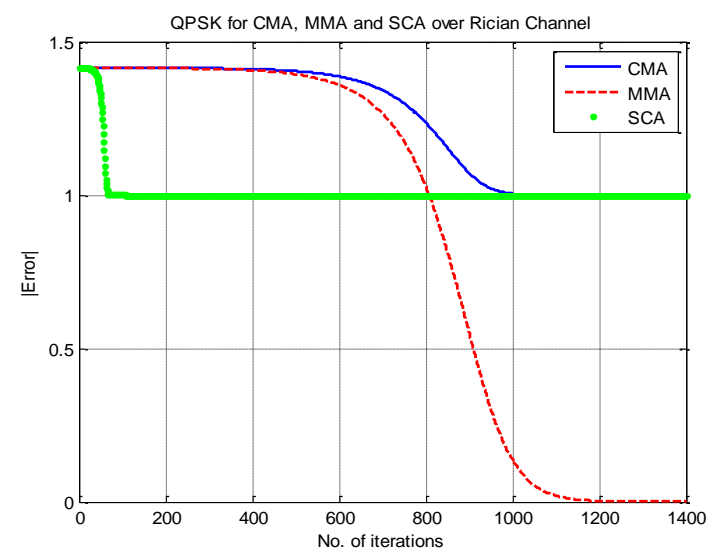

Figure 5: QPSK for CMA, SCA and MMA over Rician Channel

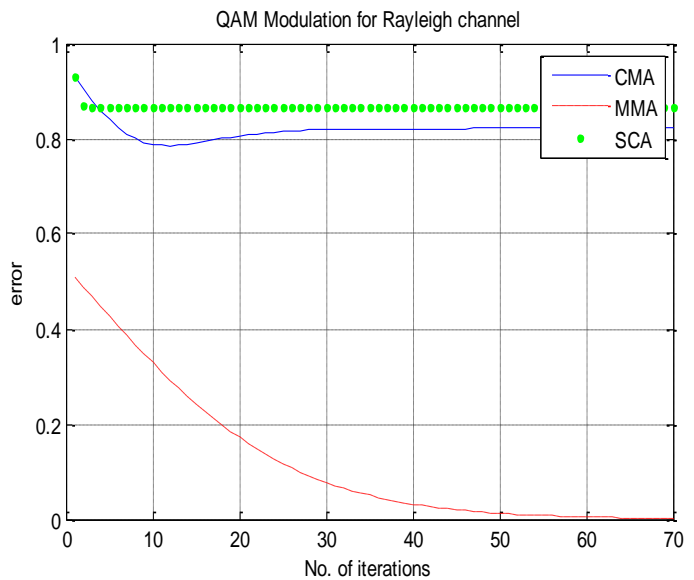

Figure 6: QAM for CMA, SCA and MMA over Rayleigh Channel

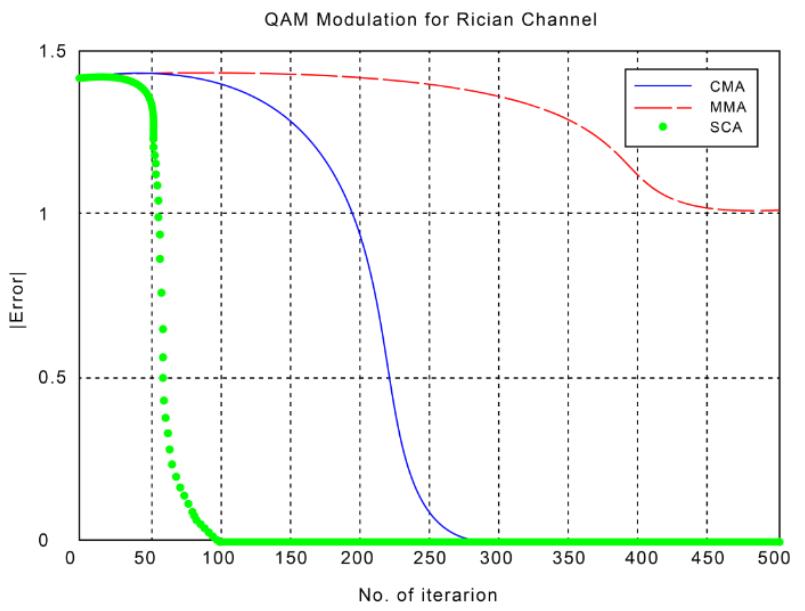

Figure 7: QAM for CMA, SCA and MMA over Rician Channel

In the following figures, simulations using two modulations techniques with a single access technique over two wireless channels are shown. 


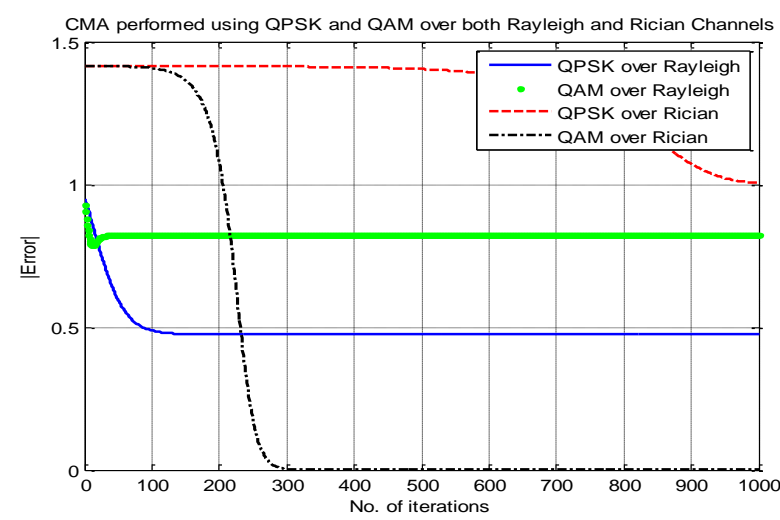

Figure 8: CMA performed using QPSK \& QAM over both Rayleigh \& Rician Channel

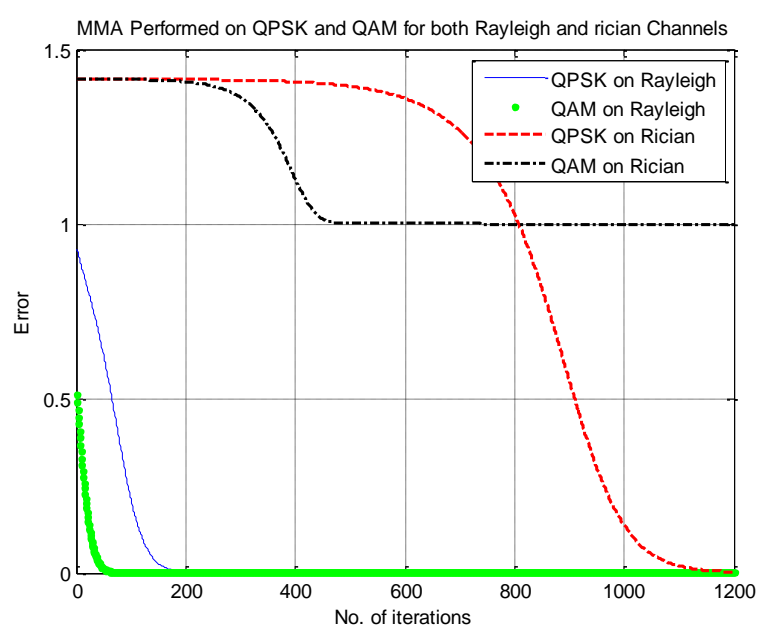

Figure 9: MMA performance on QPSK \& QAM for both Rayleigh \& Rician Channel

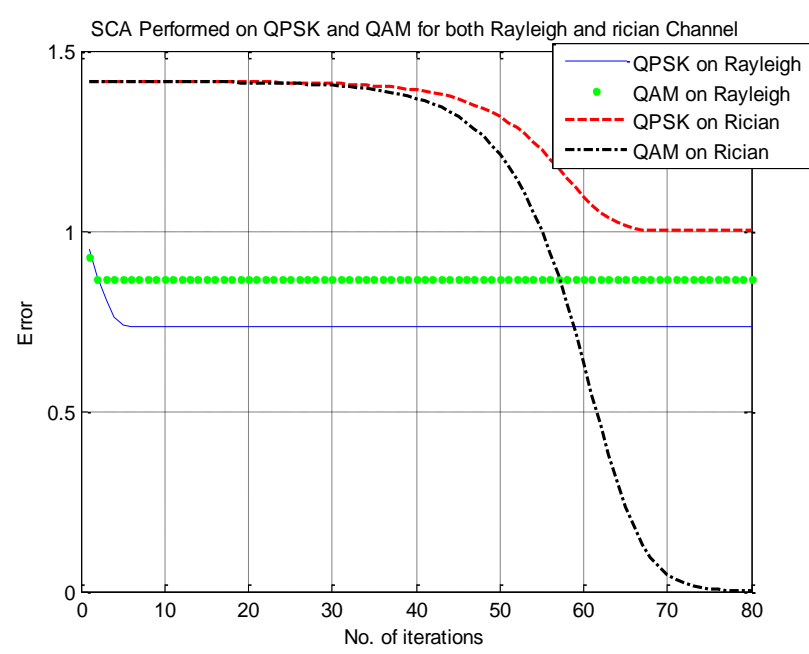

Figure 10: SCA Performed on QPSK \& QAM on both Rayleigh \& Rician Channel

Based on the simulation results, statistics results are tabulated in the followings.

\begin{tabular}{|c|c|c|c|c|c|c|}
\hline \multirow{2}{*}{ Algorithm } & \multicolumn{3}{|c|}{ Rayleigh Channel } & \multicolumn{3}{c|}{ Rician Channel } \\
\cline { 2 - 7 } & BPSK & QPSK & QAM & BPSK & QPSK & QAM \\
\hline CMA & 1000 & 1000 & 300 & 1000 & 1000 & 2950 \\
\hline MMA & 1800 & 1800 & 600 & 11600 & 11600 & 4800 \\
\hline SCA & 50 & 50 & 20 & 650 & 650 & 750 \\
\hline
\end{tabular}

Table 1: for convergence of CMA, MMA and SCA using BPSK, QPSK and QAM separately for Rayleigh and Rician channels for step size .001

\begin{tabular}{|c|c|c|c|c|c|c|}
\hline \multirow{2}{*}{ Algorithm } & \multicolumn{3}{|c|}{$\begin{array}{l}\text { Rayleigh Channel } \\
\text { (Chan1) }\end{array}$} & \multicolumn{3}{|c|}{ Rician Channel(Chan1) } \\
\hline & BPSK & QPSK & QAM & BPSK & QPSK & QAM \\
\hline CMA & 100 & 100 & 30 & 1000 & 1000 & 300 \\
\hline MMA & 180 & 180 & 62 & 1200 & 1200 & 490 \\
\hline SCA & 6 & 6 & 3 & 75 & 75 & 90 \\
\hline
\end{tabular}

Table 2: convergence of CMA, MMA and SCA using BPSK, QPSK and QAM separately for Rayleigh (Chan1) and Rician (Chan1) Channels for step size .01

\begin{tabular}{|l|l|l|l|l|l|l|}
\hline \multirow{2}{*}{ Algorithm } & \multicolumn{2}{|l|}{$\begin{array}{l}\text { Rayleigh Channel } \\
\text { (Chan2) }\end{array}$} & \multicolumn{2}{l|}{$\begin{array}{l}\text { Rician Channel } \\
\text { (Chan1) }\end{array}$} & \multicolumn{2}{l|}{} \\
\cline { 2 - 7 } & BPSK & QPSK & QAM & BPSK & QPSK & QAM \\
\hline CMA & 240 & 240 & 60 & 1000 & 1000 & 300 \\
\hline MMA & 400 & 400 & 145 & 1200 & 1200 & 490 \\
\hline SCA & 13 & 13 & 6 & 70 & 70 & 75 \\
\hline
\end{tabular}

Table 3: convergence of CMA, MMA and SCA using BPSK, QPSK and QAM separately for Rayleigh (chan2) and Rician (Chan1) channels for step size .01

\begin{tabular}{|l|l|l|l|l|l|l|}
\hline \multirow{2}{*}{ Algorithm } & \multicolumn{2}{|l|}{$\begin{array}{l}\text { Rayleigh Channel } \\
\text { (Chan1) }\end{array}$} & \multicolumn{4}{l|}{$\begin{array}{l}\text { Rayleigh Channel } \\
\text { (Chan2) }\end{array}$} \\
& \multicolumn{2}{|l|}{} & \multicolumn{3}{l|}{} \\
\cline { 2 - 7 } & BPSK & QPSK & QAM & BPSK & QPSK & QAM \\
\hline CMA & 100 & 100 & 30 & 240 & 240 & 60 \\
\hline MMA & 180 & 180 & 62 & 400 & 400 & 145 \\
\hline SCA & 6 & 6 & 3 & 13 & 13 & 6 \\
\hline
\end{tabular}

Table 4: Convergence of CMA, MMA and SCA using BPSK, QPSK and QAM separately for Rayleigh channels chan1 and chan2 for step size .01 


\section{CONCLUSION}

Based on the figures 1-10, that is conclude about the signal convergence analysis with respect to their SNR, keeping in view the modulation techniques and wireless channel. The graphs revealed that when increase the order of the modulated signal, performance decreases and there comes a time when PSK variants must be replaced with QAM variants. Note that blind equalization designs do not require training signals so spectral efficiency is naturally improved and bandwidth can be saved. The training signal method is a new concept that should be studied to increase better communication realization.

From Table 1 to table 4, following points are concluded based on the measurements:

- On all algorithms convergence rate of QPSK and BPSK is same.

- Rayleigh channel converges faster than Rician channel.

- Convergence rate decreases by reducing the number of channel coefficients.

- Square contour algorithm has greater rate of convergence than constant modulus algorithm and multi modulus algorithm.

- Multi modulus algorithm has slowest rate of convergence than both constant modulus and square contour algorithms.

- $\quad$ SCA and CMA perform better only for QAM over Rician channel (where MMA gives worst performance). But comparing SCA and CMA, SCA is fast in convergence.

- $\quad$ SCA and CMA performs better only for QAM over Rician channel (where MMA gives worst performance). But comparing SCA and CMA, SCA is fast in convergence.

- MMA gives superior performance over all modulation techniques and channels except QAM over Rician channel.

\section{FUTURE WORK}

Novel blind channel equalizers are being developed which are new to traditional works that have been done in this field. The problems in higher order QAM as discussed in previous chapters need to be studied. These novel methods should be studied and comparative analysis of those techniques should be made with traditional ones. Arbitrary high order QAM is one of the novel techniques that is being used for adaptability of the QAM module but this makes system is costlier beyond implementation for ordinary communications.

Performance of blind equalization can be enhanced by using algorithms that deal with errors more specifically. The factor upon which adaptive algorithm depends is the cost function. Error equation is used as definition of the cost function so algorithm dealing with better equations can be used in future to further enhance the performance of the equalization process.

Tracking performance of the equalizers can be improved by applying predictor at the equalizer end. Predictor at output of the equalizer adjusts the fading gain of the channel. A linear compensation for channel gain may provide better performance as well. Another future trend in equalization process may get started by combining the equalization process and error equations which will both deal with the tracking issues and error reduction in the data. The proposed compensation may perhaps result in a more analytically tractable system than the system used in this work.

Conventional equalizations minimizing mean-square error usually need a training sequence associated with the data sequence. So, in the future work Blind Equalization based on Modified Constant Modulus Algorithm could be introduced which improves the data rate and quality. The commonly used adaptive algorithm for blind channel equalization is the Constant Modulus Algorithm (CMA), which uses the constant modularity of the signal as the desired property. The design displays reasonable convergence rate and extensive consistent state mean square error and the stage blind nature in correlation with the calculations utilized as a part of traditional information supported adjustment plans.

\section{ACKNOWLEDGEMENT}

Many people have made possible contribution to my work on this research in different ways. It is a pleasure to convey my gratitude and to them all in my humble acknowledgement. Foremost the support, guidance and encouragement by my supervisor Dr. Aftab Hussain Bangash, Mr. Asad Ehsan \& Mr. Mudassar Nawaz for their guidance and support at critical stages. Their professional vision and technical skills always proved helpful in problem solving and addressing issue.

\section{REFERENCES}

[1] Hodges, M.R., 1990. The GSM radio interface. British Telecom Technology Journal, 8(1), pp.31-43.

[2] Sato, Y., 1975. A method of self-recovering equalization for multilevel amplitude-modulation systems. IEEE Transactions on communications, 23(6), pp.679-682.

[3] Abrar, S., Zerguine, A. and Nandi, A.K., 2012. Adaptive blind channel equalization. Digital Communication, InTech Publishers, Rijeka, Croatia, pp.93-118.

[4] Jones, D.L., 1995, October. A normalized constantmodulus algorithm. In Signals, Systems and Computers, 1995. 1995 Conference Record of the Twenty-Ninth Asilomar Conference on (Vol. 1, pp. 694-697). IEEE.

[5] Gitlin, R. and Mazo, J., 1973. Comparison of some cost functions for automatic equalization. IEEE Transactions on communications, 21(3), pp.233-237.

[6] Sato, Y., 1975. A method of self-recovering equalization for multilevel amplitude-modulation systems. IEEE Transactions on communications,23(6), pp.679-682.

[7] Godard, D., 1980. Self-recovering equalization and carrier tracking in two-dimensional data communication systems. IEEE transactions on communications, 28(11), pp.1867-1875.

[8] Wesolowski, K., 1987. Self-recovering adaptive equalization algorithms for digital radio and voiceband data modems. In Proc. European Conf. Circuit Theory and Design (pp. 19-24).

[9] Ruget, G. (1980a). Analysis of stochastic approximation schemes with discontinous and dependent forcing terms with applications to data communications algorithms, IEEE Trans. Automat. Contr. 25(12): 1042-1058

[10] Yuan, J.T. and Tsai, K.D., 2005. Analysis of multi modulus blind equalization algorithm in QAM communication systems. IEEE Transactions on Communications, 53(9), pp.1427-1431. 
[11] Li, Y. and Ding, Z., 1995. Convergence analysis of finite length blind adaptive equalizers. IEEE Transactions on Signal Processing, 43(9), pp.2120-2129.

[12] Liu, D. and Tong, L., 1999. An analysis of constant modulus algorithm for array signal processing. Signal Processing, 73(1), pp.81-104.

[13] Zhang, L., Montage Technology Group, Ltd, 2009. Hybrid modulus blind equalization for quadrature amplitude modulation (QAM) receivers. U.S. Patent $7,599,449$.

[14] Ram Babu, T. and Kumar, P.R., 2010. Blind Equalization using Constant Modulus Algorithm and Multi-Modulus Algorithm in Wireless Communication Systems. International Journal of Computer Applications, 1(3), pp.50-55.

[15] Abrar, S. and Nandi, A.K., 2008, August. Normalized constant modulus algorithm for blind channel equalization. In Signal Processing Conference, 2008 16th European (pp. 1-5). IEEE.

[16] Ding, Z. \& Li, Y. (2001). Blind Equalization and Identification, Marcel Dekker Inc., New York.

[17] Moshirian, Sanaz, Soheil Ghadami, and Mohammad Havaei. "Blind Channel Equalization." arXiv preprint arXiv: 1208.2205 (2012).

[18] D. L. Jones, “A normalized constant-modulus algorithm", Proceedings of 29th Asilomar Conference on Signals, Systems and Computers, Pacific Grove, CA,USA, vol.2, pp.694-697, October 1999

[19] Haykin, S. (1996). Adaptive Filtering Theory, PrenticeHall.

[20] Lucky, R. (1966). Techniques for adaptive equalization of digital communication systems, The Bell Systems Technical Journal pp. 255-286.
[21] Widrow, B. \& Hoff, M.E. (1960). Adaptive switching circuits, Proc. IRE WESCON Conf. Rec. pp. 96-104.

[22] Allen, J. \& Mazo, J. (1973). Comparison of some cost functions for automatic equalization, IEEE Trans. Commun. 21(3): 233-237.

[23] Sato, Y. (1975). A method of self-recovering equalization for multilevel amplitude modulation systems, IEEE Trans. Commun. 23(6): 679-682.

[24] Godard, D. (1980). Self-recovering equalization and carrier tracking in two-dimensional data communications systems, IEEE Trans. Commun. 28(11): 1867-1875

[25] Wesolowski, K. (1987). Self-recovering adaptive equalization algorithms for digital radio and voiceband data modems, Proc. European Conf. Circuit Theory and Design pp. 19-24.

[26] Shafayat, Azzedine, "Adaptive Blind Channel Equalization", Published online 07, March, 2012 Published in print edition March, 2012

[27] Bellini, S. (1986). Bussgang techniques for blind equalization, Proc. IEEE GLOBECOM'86 pp. 16341640.

[28] Ruget, G. (1980a). Analysis of stochastic approximation schemes with discontinous and dependent forcing terms with applications to data communications algorithms, IEEE Trans. Automat. Contr. 25(12): 1042-1058

[29] Shalvi, O. \& Weinstein, E. (1990). New criteria for blind equalization of non-minimum phase systems, IEEE Trans. Inf. Theory 36(2): 312-321.

[30] K Suthendran, Performance Comparison of Blind Equalization Algorithms for Wireless Communication, International Journal of Computer Applications (0975 8887), Volume 85 - No 13, January 2014. 